\section{Safety and feasibility of ultrasound- guided insertion of peripherally inserted central catheter performed by an intensive care trainee}

Yongwoo Lee, MD'; Jeong-Am Ryu, MD, PhD, ; Yong Oh Kim, MD²; Eunmi Gil, MD ${ }^{2,4}$; Young-Mok Song, MD, PhD'

${ }^{1}$ Department of Neurology, Dankook University Hospital, Dankook University College of Medicine, Cheonan, Republic of Korea

${ }^{2}$ Department of Critical Care Medicine, Samsung Medical Center, Sungkyunkwan University School of Medicine, Seoul, Republic of Korea

${ }^{3}$ Department of Neurosurgery, Samsung Medical Center, Sungkyunkwan University School of Medicine, Seoul, Republic of Korea

${ }^{4}$ Department of Surgery, Samsung Medical Center, Sungkyunkwan University School of Medicine, Seoul, Republic of Korea

\section{Care}

\section{ORIGINAL ARTICLE}

Received: November 22, 2019

Revised: January 2, 2020

Accepted: January 18, 2020

Corresponding Author:

Young-Mok Song, MD, PhD

Department of Neurology, Dankook

University Hospital, Dankook University

College of Medicine, 201 Manghyang-

ro, Dongnam-gu, Cheonan 31116,

Republic of Korea

Tel: +82-41-550-6577

Fax: +82-41-550-0524

E-mail:ymsong@dkuh.co.kr

Background: We investigated the safety and feasibility of ultrasound-guided peripherally inserted central venous catheter (PICC) placements performed by intensive care medical trainees in comparison to PICC placements performed by intensivists.

Methods: This was a retrospective and observational study of adult patients who underwent PICC placement and were admitted to the intensive care unit (ICU) between July 2013 and March 2018. Ultrasound-guided PICC was performed at the bedside by an intensivist or intensive care medical trainee if intrahospital transport was inappropriate. The primary endpoint was PICC-induced complications. The secondary endpoint was initial success of PICC.

Results: A total of 209 patients underwent PICC placement during the study period. There were no significant differences in age, sex, body mass index, comorbidities, causes of ICU admission, or severity scores between the trainee-led PICC and intensivist-led PICC groups. Difficult venous access (42.6\%) and requirement for central line infusion (39.2\%) were the most common reasons for PICC placement. The basilic vein (62.2\%) was the most common target vein among patients who underwent PICC. There were no significant differences in complications between the two groups $(P=0.473)$. In addition, the initial success rate and procedural time were similar between the two groups $(P=0.108$ and $P=0.076$, respectively). There were no insertional injuries and moderate or severe bleeding in patients with PICC.

Conclusion: Ultrasound-guided PICC placement by an intensive care medical trainee may be safe and feasible compared to PICC placement by an intensivist.

Keywords: Peripherally inserted central venous catheter; Ultrasound; Specialist; Trainee

\section{INTRODUCTION}

Peripherally inserted central venous catheters (PICCs) are commonly used as an alternative to central venous catheters in critical- ly ill patients $[1,2]$. There are many theoretical advantages to using PICC such as easy placement and a lower rate of complications [1-5]. In the past, interventional radiologists inserted PICCs in the interventional radiology suite under fluoroscopic guidance

(C) 2020 The Korean Neurocritical Care Society

This is an Open Access article distributed under the terms of the Creative Commons Attribution Non-Commercial License (http://creativecommons.org/licenses/by-nc/4.0/) which permits unrestricted noncommercial use, distribution, and reproduction in any medium, provided the original work is properly cited. 
$[2,6,7]$. However, serious adverse events may occur during intrahospital transport of critically ill patients due to respiratory failure or hemodynamic instability [8-10]. Therefore, PICC may be implemented at the bedside by intensivists for critically ill patients who have transport risks [8]. In addition, ultrasound-guided PICC placements performed by intensivists have been gradually increasing due to its safety and ease-of-use [11].

A subspecialty training program was recently established in the field of intensive care medicine in Korea [12]. Therefore, intensive care medical trainees have been recently trained in and implemented PICC placements. However, there is limited data on the safety and feasibility of ultrasound-guided PICC placement performed by trainees of intensive care medicine. Therefore, the objective of this study was to investigate the safety and feasibility of ultrasound-guided PICC placements performed by intensive care medical trainees compared to PICC placements performed by intensivists.

\section{METHODS}

This was a retrospective and observational study of adult patients admitted to the intensive care units (ICUs) at Samsung Medical Center between July 2013 and March 2018. This study was approved by the Institutional Review Board of Samsung Medical Center (SMC 2018-09-011). The requirement for informed consent was waived due to its retrospective nature.

\section{Study population}

Adult patients who underwent ultrasound-guided PICC performed by an intensive care medical trainee or an intensivist during their ICU stay were included in the study. Of these patients, patients younger than 18 years of age, those with insufficient medical records, and those discharged before 14 days after PICC placement were excluded. A total of 209 patients with PICC placement were analyzed in this study (Fig. 1). To assess the safety and feasibility of PICC performed by subspecialty trainees in critical care medicine, postprocedural outcomes were compared between trainee-led and intensivist-led procedures.

\section{Definitions and outcomes}

We retrospectively reviewed all placements of PICCs in the ICU during the study period. The illness severity on ICU admission was estimated by the Simplified Acute Physiology Score 3 (SAPS 3) and Sequential Organ Failure Assessment (SOFA) systems. Central line-associated bloodstream infections (CLABSIs) were defined as an infection resulting from the same bacteria as confirmed by line and blood cultures $[4,11,13,14]$. CLABSIs were also identified in collaboration with the Infection Prevention and Control Team of Samsung Medical Center who monitors for CLABSIs [11]. Compression ultrasonography and duplex Doppler were performed only when the patient had clinical signs and symptoms suggesting venous occlusion such as a swelled arm, unexplained local pain, or malfunctioned PICC $[1,11]$.

In this study, the primary outcome was PICC-induced complications. The secondary outcome was initial success of central line placement.

\section{Procedure}

An intensivist determined the PICC placement in critically ill patients. The ultrasound-guided PICC was preferred at the bedside for patients with hemodynamic instability, on mechanical ventilation, or those who were critically ill. In this study, all PICCs were inserted as an elective procedure. Indications for placement of PICC included the need for a central line for parenteral nutrition, infusion of drugs requiring a central line, need for frequent blood sampling, or difficult venous access $[1,11]$. Contraindications to PICC placement were small deep veins of the arm (diameter of target vein $<3 \mathrm{~mm}$ ), local contraindications due to specific arm conditions such as skin infection, burns, or an arteriovenous fistula for renal failure [11]. Obesity or severe edema were not considered contraindications for PICC placement. We used 5 Fr single-lumen silastic catheters or 5 Fr dual-lumen Turbo-Ject Power-Injectable PICCs (Cook, Bloomington, MN, USA), 5 Fr triple-lumen PowerPICC Catheters (Bard Access Systems, Salt Lake City, UT, USA), or 6 Fr dual-lumen Vaxel PICCs with PASV

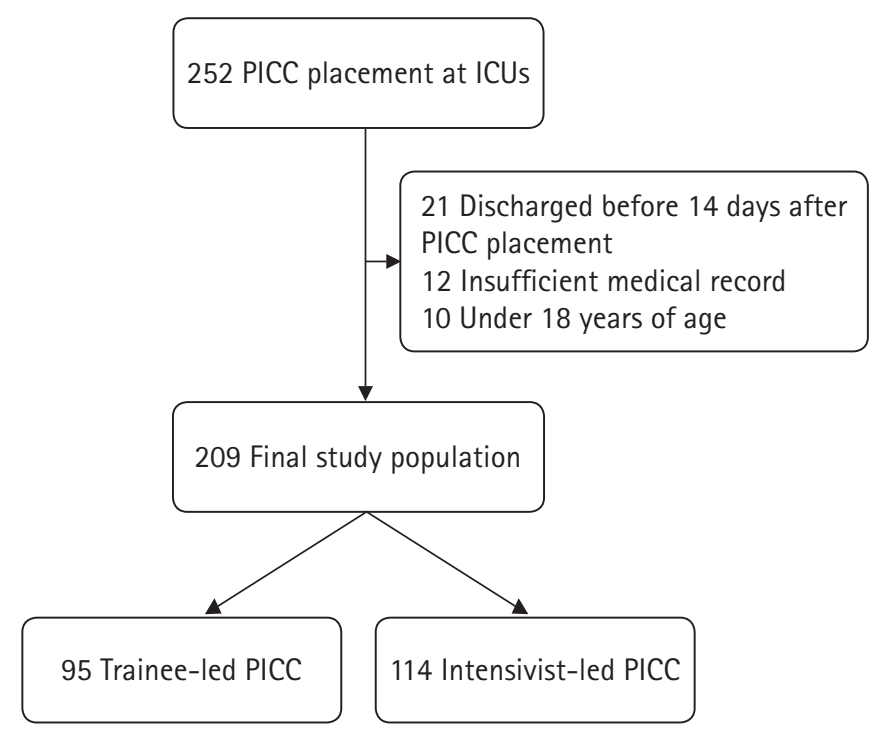

Fig. 1. Study flow chart. PICC, peripherally inserted central catheter; ICU, intensive care unit. 
Valves (Navilyst, Marlborough, MA, USA) [11]. PICC placement was performed by an intensivists or intensive care medical trainee. After trainees had seen the procedure three times, intensivists supervised their procedures two or three times before they performed the PICC placement independently. In the supine position, the patient's arm was abducted and externally rotated. Usually, the target vein is either the basilic, brachial, or cephalic vein, and a tourniquet is sometimes applied to help find the target veins. The PICC placement was performed by an ultrasound-guided puncture of the deep veins in the upper midarm using the microintroducer technique. A standard 5 to $10 \mathrm{MHz}$ linear ultrasound probe was used. The optimal length was determined for placement of the catheter tip in the cavoatrial junction, which is commonly done by measuring the distance from the site of insertion through the humeral head to the sternal notch, and down to the 3rd intercostal space. Maximal barrier precautions were consistently used during the procedure [11]. Ultrasound was used to anesthetize the skin and reidentify veins. After the ultrasound-guided puncture of the deep vein, the syringe was removed and the guide wire was advanced through the needle. The needle was removed and the wire placement in the vein was confirmed using ultrasound. Using a scalpel, a small nick at the insertion site was created to accommodate the dilator. The dilator and introducer were inserted over the guide wire. After removal of the guide wire and dilator, only the introducer was left in place. Finally, the catheter was inserted through the introducer and advanced to the predetermined length before the introducer was removed. The target veins and procedure are shown in Fig. 2. The correct position of the tip of the catheter was verified by chest radiographs. Malposition was defined as a catheter tip that was not located at the cavoatrial junction
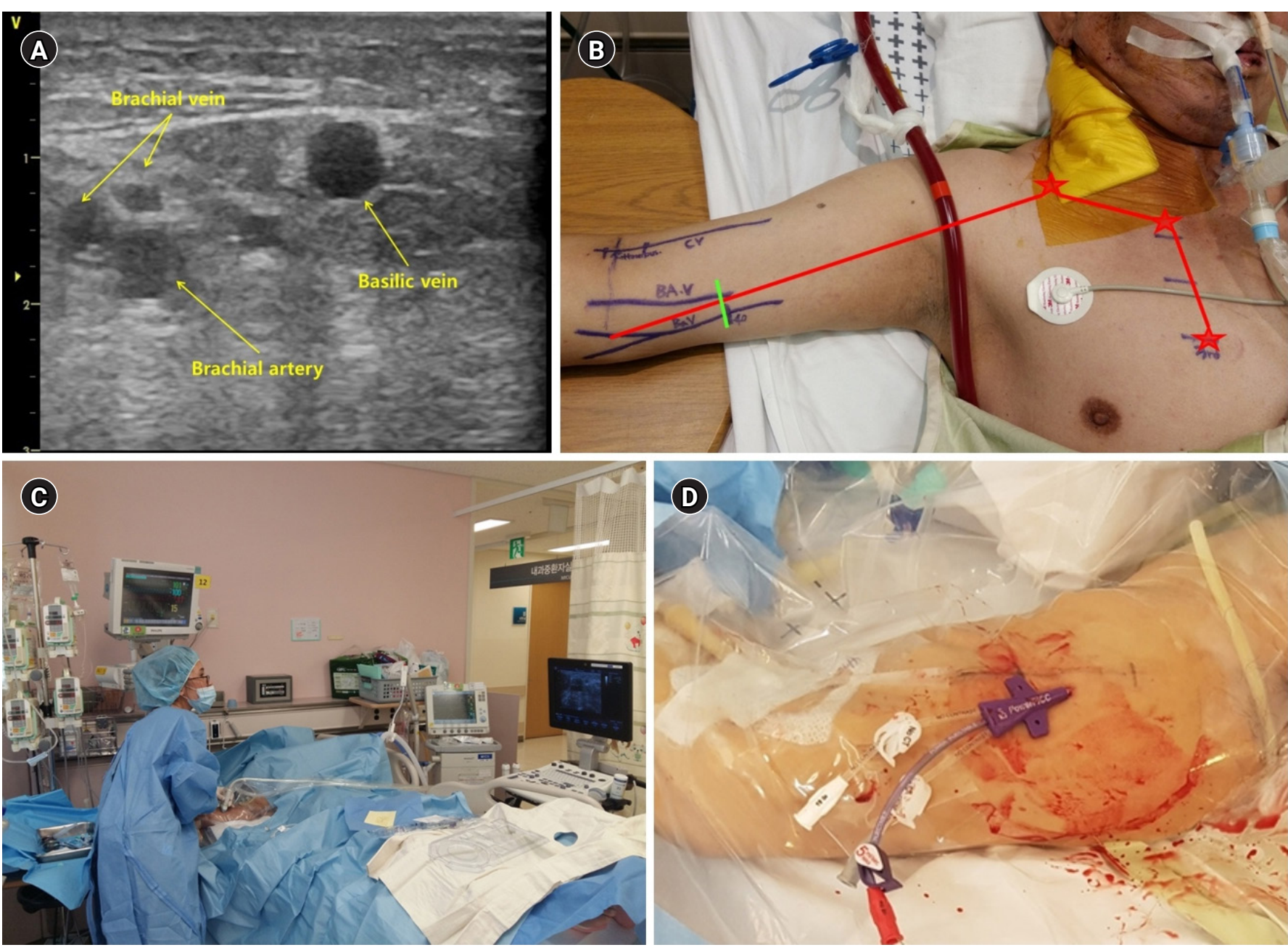

Fig. 2. (A) Target veins and procedure of the ultrasound-guided insertion of the peripherally inserted central catheter (PICC). Target veins were accessed by ultrasound. The cephalic vein is not shown in this ultrasound image. (B) The optimal length of the inserted PICC is measured from the site of insertion through the humeral head to the sternal notch, and down to the 3rd intercostal space. (C) The PICC is being inserted with the ultrasound-guided method in the intensive care unit (Eunmi Gil). (D) Finally, the PICC insertion is completed. 
in chest radiographs. Dressing changes occurred every 7 days, or if soiled in all placements [11].

\section{Statistical analyses}

All data are presented as mean \pm standard deviation for continuous variables and numbers (percentages) for categorical variables. We compared data using Student's $t$ test for continuous variables and chi-square test or Fisher's exact test for categorical variables. All tests were two-sided and $P$ values $<0.05$ were considered statistically significant. Data were analyzed using IBM SPSS statistics ver. 20 (IBM, Armonk, NY, USA).

\section{RESULTS}

\section{Baseline characteristics}

A total of 209 patients underwent PICC placements during their ICU stay. The mean age of the patients with PICC was 59.3 \pm 15.9 years. Of 209 patients, 116 (55.5\%) were males. Hypertension (54.5\%) and malignancies (53.6\%) were the most common comorbidities among patients who underwent PICC placement. Respiratory failure (27.3\%) and sepsis (23.4\%) were the most common causes of ICU admission. There were no significant differences in age, gender, body mass index, comorbidities, causes of ICU admission, or severity scores of illness on ICU admission be- tween the two groups (Table 1).

\section{Procedural characteristics}

Difficult venous access (42.6\%) and requirement for central line infusion (39.2\%) were the most common reasons for PICC placement. Almost all patients (92.8\%) used a mechanical ventilator and 70 patients $(33.5 \%)$ had hemodynamic instability. Renal replacement therapy was more frequently used in the trainee group compared to the intensivist group. The basilic vein (62.2\%) and the brachial vein $(20.6 \%)$ were the most common target veins among patients who underwent PICC placement. Activated partial thromboplastin time was prolonged in the trainee-led PICC group compared to the intensivist-led PICC group. There were no significant differences in reasons for PICC placement, use of anticoagulant and antiplatelet agent, insertional veins, platelet count, and international normalized ratio between the two groups (Table 2). The procedure times of the intensive medical trainees and intensivists according to procedure number are shown Fig. 3. Although the procedure times of intensive medical trainees decreased after three or four procedures (Fig. 3A), those of intensivists according to procedure number were similar (Fig. 3B).

\section{Clinical outcomes}

There were no significant differences in complication between

Table 1. Baseline characteristics

\begin{tabular}{|c|c|c|c|}
\hline Characteristic & Insertion by intensivist $(\mathrm{n}=95)$ & Insertion by trainee $(n=114)$ & $P$ value \\
\hline Age (yr) & $58.4 \pm 15.6$ & $60.1 \pm 16.2$ & 0.444 \\
\hline Male sex & $52(54.7)$ & $64(56.1)$ & 0.949 \\
\hline BMI $\left(\mathrm{kg} / \mathrm{m}^{2}\right)$ & $23.3 \pm 4.5$ & $22.5 \pm 4.5$ & 0.222 \\
\hline Obese $\left(\mathrm{BMI}>30 \mathrm{~kg} / \mathrm{m}^{2}\right)$ & $6(6.4)$ & $4(3.8)$ & 0.522 \\
\hline \multicolumn{4}{|l|}{ Comorbidities } \\
\hline Hypertension & $55(57.9)$ & $59(51.8)$ & 0.454 \\
\hline Malignancy & $53(55.8)$ & $59(51.8)$ & 0.658 \\
\hline Diabetes mellitus & $36(37.9)$ & $45(39.5)$ & 0.928 \\
\hline Chronic kidney disease & $22(23.2)$ & $26(22.8)$ & 0.999 \\
\hline Chronic liver disease & $7(7.4)$ & $16(14.0)$ & 0.190 \\
\hline Ischemic heart disease & $7(7.4)$ & $13(11.4)$ & 0.452 \\
\hline Cause of ICU admission & & & 0.242 \\
\hline Respiratory failure & $24(25.3)$ & $33(28.9)$ & \\
\hline Sepsis & $21(22.1)$ & $28(24.6)$ & \\
\hline Cardiovascular problems & $20(21.1)$ & $28(24.6)$ & \\
\hline Neurological abnormalities & $27(28.4)$ & $18(15.8)$ & \\
\hline Other & $3(3.2)$ & $7(6.1)$ & \\
\hline SOFA score & $7.7 \pm 4.5$ & $8.5 \pm 4.1$ & 0.267 \\
\hline SAPS 3 & $36.1 \pm 14.6$ & $10.6 \pm 15.0$ & 0.059 \\
\hline
\end{tabular}

Values are presented as mean \pm standard deviation or number (\%).

BMI, body mass index; ICU, intensive care unit; SOFA, Sequential Organ Failure Assessment; SAPS, Simplified Acute Physiology Score. 
the two groups $(P=0.473)$. There were five cases of CLABSI (2.4\%) and three cases of symptomatic PICC-related venous thrombosis $(1.4 \%)$ in all patients who underwent PICC placement. However, there were no insertional injuries and moderate or severe bleeding in patients with PICC (Table 3). In addition, there were no significant differences in the rate of initial success and procedure time between the two groups $(P=0.108$ and
$P=0.076$, respectively)

\section{DISCUSSION}

In this study, we investigated the safety and feasibility of ultrasound-guided PICC placement performed by intensive care medical trainees compared to PICC placements performed by inten-

Table 2. Procedural characteristics of peripherally inserted central venous catheter line placement

\begin{tabular}{lccc}
\hline Characteristic & Insertion by intensivist $(n=95)$ & Insertion by trainee $(n=114)$ & $P$ value \\
\hline Reason for PICC insertion & & & 0.799 \\
Difficult venous access & $39(41.1)$ & $50(43.9)$ & \\
For infusion of drugs requiring a central line & $38(40.0)$ & $44(38.6)$ & $11(9.6)$ \\
Parenteral nutrition & $13(13.7)$ & $6(5.3)$ & 0.999 \\
Frequent blood sampling & $4(4.2)$ & $3(2.6)$ & 0.569 \\
Other & $1(1.1)$ & $40(35.1)$ & 0.212 \\
Anticoagulation & $33(34.7)$ & $8(7.0)$ & 0.025 \\
Use of antiplatelet agent & $4(4.2)$ & $103(90.4)$ & 0.156 \\
Use of mechanical ventilator & $91(95.8)$ & $57(50.0)$ & 0.654 \\
Use of renal replacement therapy & $32(33.7)$ & $43(37.7)$ & \\
Use of vasopressor or hypotension & $27(28.4)$ & $70(61.4)$ & $22(19.3)$ \\
Insertional site & & $22(19.3)$ & \\
Basilic vein & $60(63.2)$ & & \\
Brachial vein & $21(22.1)$ & $158.5 \pm 126.2$ & 0.068 \\
Cephalic vein & $14(14.7)$ & $1.7 \pm 1.1$ & 0.154 \\
Laboratory results of coagulation on the day of PICC & $191.0 \pm 128.3$ & $52.1 \pm 16.1$ & 0.049 \\
Platelet count $\left.\times 10^{3} / \mu \mathrm{L}\right)$ & $1.4 \pm 0.9$ & & \\
INR & $47.4 \pm 15.4$ & & \\
aPTT (sec)
\end{tabular}

Values are presented as number (\%) or mean \pm standard deviation.

PICC, peripherally inserted central catheter; INR, international normalized ratio; aPT, activated partial thromboplastin time.
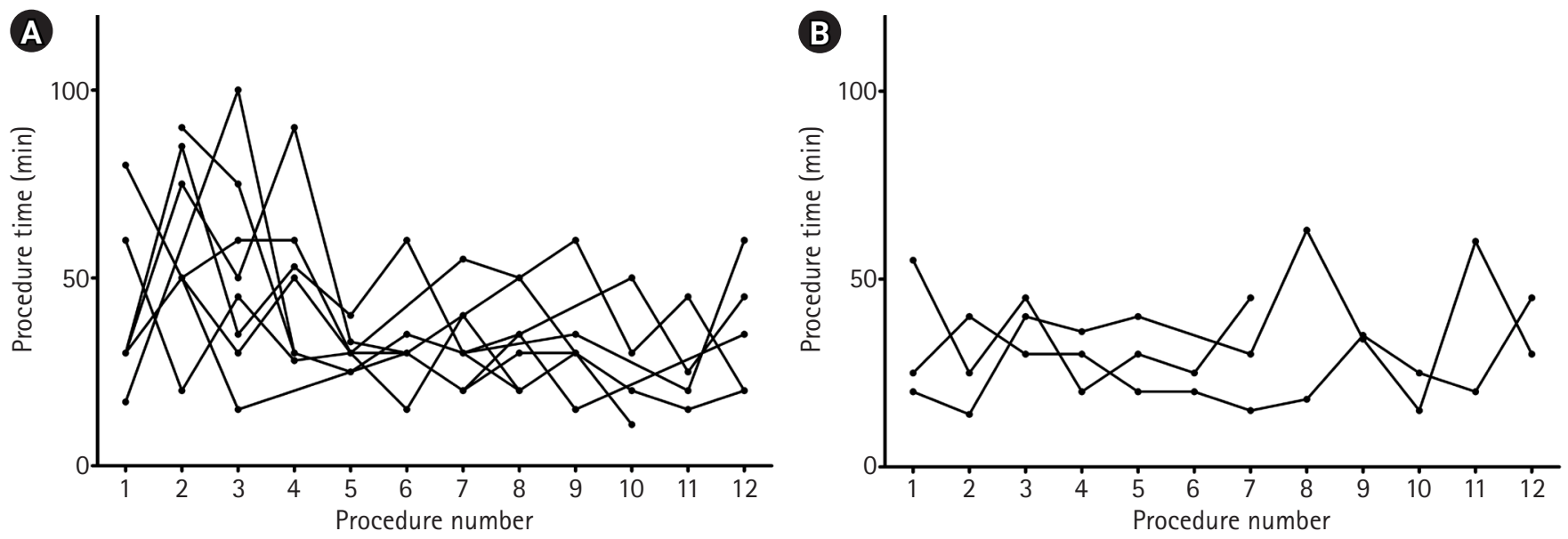

Fig. 3. The procedure times of $(A)$ intensive medical trainees and $(B)$ intensivists according to procedure number. $(A)$ Although the procedure times of intensive medical trainees were decreased after three or four procedures, $(\mathrm{B})$ those of intensivist according to procedure number were similar. 
Table 3. Clinical outcomes

\begin{tabular}{|c|c|c|c|}
\hline Characteristic & Insertion by intensivist $(n=95)$ & Insertion by trainee $(n=114)$ & $P$ value \\
\hline \multicolumn{4}{|l|}{ Procedural data } \\
\hline Initial success of PICC & $87(91.6)$ & $96(84.2)$ & 0.108 \\
\hline Malposition \& reinsertion & $8(8.4)$ & $6(5.3)$ & 0.363 \\
\hline Procedure time (min) & $32.3 \pm 19.9$ & $37.6 \pm 20.8$ & 0.076 \\
\hline Duration of using PICC (day) & $80 \pm 20.1$ & $102 \pm 32.4$ & 0.112 \\
\hline Complication & $5(5.3)$ & $3(2.6)$ & 0.473 \\
\hline CLABSI & $3(3.2)$ & $2(1.8)$ & 0.661 \\
\hline Symptomatic PICC-related venous thrombosis & $2(2.1)$ & $1(0.9)$ & 0.179 \\
\hline Insertional injury & 0 & 0 & NS \\
\hline Moderate or severe bleeding and hematoma & 0 & 0 & NS \\
\hline Cause of removal & & & 0.723 \\
\hline Unnecessary & $58(61.1)$ & 77 (67.5) & \\
\hline Malfunction & $22(23.2)$ & $21(18.4)$ & \\
\hline Fever & $9(9.5)$ & $8(7.0)$ & \\
\hline Self-removal & $6(6.3)$ & $8(7.0)$ & \\
\hline
\end{tabular}

Values are presented as number (\%) or mean \pm standard deviation.

PICC, peripherally inserted central catheter; CLABSI, central line-associated bloodstream infection; NS, not significant with $P>0.05$.

sivists. We report multiple major findings in this study. First, there were no significant differences in complications associated with PICC insertion between the two groups. In addition, there were no insertional injuries and severe bleeding in both groups. The incidence rates of CLABSI and symptomatic PICC-related venous thrombosis were low in patients with PICC. Second, the rate of initial success and procedural time were similar between the two groups. In addition, the procedure times of trainees decreased after three or four procedures. Third, PICC placement was performed in patients with intrahospital transport risks due to mechanical ventilator or hemodynamic instability. Fourthly, difficult venous access and requirement for central line infusion were the most common reasons for PICC placement. Overall, we show that ultrasound-guided PICC placement is a well-established procedure and easy to learn by an intensive care medical trainee.

Critically ill patients often need central venous access using either a central venous catheter or PICC due to various reasons such as parenteral nutrition, long-term antibiotic therapy, frequent blood sampling, and difficult venous access $[15,16]$. PICC insertion cannot lead to hemothorax, pneumothorax, clinically significant bleeding, or hematoma, even in patients with coagulative disorders or difficult neck anatomy. In addition, PICC placement has a low risk for CRBSI compared to standard central venous catheters [1-5]. Therefore, PICC placement may be safe and useful in critically ill patients $[1,3]$.

In the past, PICC was only inserted by an interventional radiologist in the interventional radiology suite under fluoroscopic guidance PICC placements have been also performed by inter- ventional radiologists $[2,6,7]$. As this procedure is performed in the interventional radiology suite, critically ill patients should be transferred from the ICU to the interventional radiology suite. However, various complications may occur during intrahospital transport of critically ill patients from the ICU to other locations because of their respiratory failure or hemodynamic instability [810]. In addition, it may be difficult for radiologists to perform the PICC placement in the interventional radiology suite in a timely manner due to other procedures that may be ongoing in the suite. Therefore, ultrasound-guided PICC placement should be performed at the bedside in critically ill patients.

Ultrasound for vein localization and the modified Seldinger technique have been used for safe placement of PICCs at bedside $[16,17]$; these techniques could allow trainees to place the PICC easily and safely. In this study, ultrasound-guided PICC placement performed by an intensive care medical trainee was feasible compared to PICC placement performed by an intensivist. There were no significant differences in the rate of initial success and procedure times between the two groups. In addition, the intensive care medical trainees were able to safely perform the PICC placements. Additionally, the rates of PICC-related complications were similar between the two groups. A recent study also revealed that interventional radiologic placement of PICC is a well-established procedure, easy to learn by residents, and has a small learning curve [18].

This study had several limitations. This study was a retrospective review of medical records. An intensivist determined the placement of the PICC rather than following a protocol-based 
plan [11]. Therefore, a selection bias may have affected the results in this study. Additionally, there were no routine screening procedures for central line-related venous thrombosis or pulmonary thromboembolism. Finally, the statistical power of our study was limited due to the small sample size. Although it still provides valuable insight, prospective large-scale studies are needed to evaluate the safety and feasibility of ultrasound-guided PICC placement by an intensive care medical trainee.

In conclusion, ultrasound-guided PICC placement by an intensive care medical trainee may be safe and more feasible compared to PICC placement by an intensivist. Therefore, ultrasound-guided PICC placement can be performed at the bedside by an intensive care medical trainee for critically ill patients if intrahospital transport is contraindicated.

\section{ARTICLE INFORMATION}

\section{Conflict of interest}

No potential conflict of interest relevant to this article.

\section{ORCID}

Yongwoo Lee, https://orcid.org/0000-0002-0833-7754

Jeong-Am Ryu, https://orcid.org/0000-0003-1705-848X

Yong Oh Kim, https://orcid.org/0000-0001-8713-0753

Eunmi Gil, https://orcid.org/0000-0002-2002-0936

Young-Mok Song, https://orcid.org/0000-0002-1236-1271

\section{Author contributions}

Conceptualization: YL, JAR, and YMS. Data curation \& Formal analysis: YL, JAR, YOK, and EG. Visualization \& Writing-original draft: YL, JAR, and YMS. Writing-review editing: YL, JAR, YOK, EG, and YMS.

\section{Additional contributions}

We would like to thank the nursing director of the neurosurgery intensive care unit, Hye Jung Kim, who gave excellent advice and fruitful discussions. We would also like to thank all nurses of intensive care unit at Samsung Medical Center.

\section{REFERENCES}

1. Pittiruti M, Brutti A, Celentano D, Pomponi M, Biasucci DG, Annetta MG, et al. Clinical experience with power-injectable PICCs in intensive care patients. Crit Care 2012;16:R21.

2. Potet J, Arnaud FX, Thome A, Weber-Donat G, Konopacki J, Bouzad C, et al. Peripherally inserted central catheter placement in patients with coagulation disorders: a retrospective analysis.
Diagn Interv Imaging 2015;96:1147-51.

3. Pittiruti M, Hamilton H, Biffi R, MacFie J, Pertkiewicz M; ESPEN. ESPEN guidelines on parenteral nutrition: central venous catheters (access, care, diagnosis and therapy of complications). Clin Nutr 2009;28:365-77.

4. Wilson TJ, Stetler WR Jr, Fletcher JJ. Comparison of catheter-related large vein thrombosis in centrally inserted versus peripherally inserted central venous lines in the neurological intensive care unit. Clin Neurol Neurosurg 2013;115:879-82.

5. Wilson TJ, Brown DL, Meurer WJ, Stetler WR Jr, Wilkinson DA, Fletcher JJ. Risk factors associated with peripherally inserted central venous catheter-related large vein thrombosis in neurological intensive care patients. Intensive Care Med 2012; 38:272-8.

6. Donovan MS, Thomas KD, Davis DC, Hawkins K, Harris DS. Peripherally inserted central catheters: placement and use in a family practice hospital. J Am Board Fam Pract 1996;9:235-40.

7. Andrews JC, Marx MV, Williams DM, Sproat I, Walker-Andrews SC. The upper arm approach for placement of peripherally inserted central catheters for protracted venous access. AJR Am J Roentgenol 1992;158:427-9.

8. Lee DS, Park CM. Clinical feasibility of ultrasound guided placement of peripherally inserted central catheters by intensivist: preliminary report.J Acute Care Surg 2014;4:13-7.

9. Parmentier-Decrucq E, Poissy J, Favory R, Nseir S, Onimus T, Guerry MJ, et al. Adverse events during intrahospital transport of critically ill patients: incidence and risk factors. Ann Intensive Care 2013;3:10.

10. Schwebel C, Clec'h C, Magne S, Minet C, Garrouste-Orgeas M, Bonadona A, et al. Safety of intrahospital transport in ventilated critically ill patients: a multicenter cohort study. Crit Care Med 2013;41:1919-28.

11. Kim YO, Chung CR, Gil E, Park CM, Suh GY, Ryu JA. Safety and feasibility of ultrasound-guided placement of peripherally inserted central catheter performed by neurointensivist in neurosurgery intensive care unit. PLoS One 2019;14:e0217641.

12. Lee D, Chung CR, Park SB, Ryu JA, Cho J, Yang JH, et al. Safety and feasibility of percutaneous dilatational tracheostomy performed by intensive care trainee. Korean J Crit Care Med 2014; 29:64-9.

13. Maki DG, Stolz SM, Wheeler S, Mermel LA. Prevention of central venous catheter-related bloodstream infection by use of an antiseptic-impregnated catheter. A randomized, controlled trial. Ann Intern Med 1997;127:257-66.

14. Safdar N, Maki DG. Risk of catheter-related bloodstream infection with peripherally inserted central venous catheters used in hospitalized patients. Chest 2005;128:489-95. 
15. Ryder MA. Peripherally inserted central venous catheters. Nurs Clin North Am 1993;28:937-71.

16. Griffiths VR, Philpot P. Peripherally inserted central catheters (PICCs): do they have a role in the care of the critically ill patient? Intensive Crit Care Nurs 2002;18:37-47.

17. DeLemos C, Abi-Nader J, Akins PT. Use of peripherally inserted central catheters as an alternative to central catheters in neuro- critical care units. Crit Care Nurse 2011;31:70-5.

18. Rotzinger R, Gebauer B, SchnapauffD, Streitparth F, Wieners G, Grieser $C$, et al. Placement of central venous port catheters and peripherally inserted central catheters in the routine clinical setting of a radiology department: analysis of costs and intervention duration learning curve. Acta Radiol 2017;58:1468-75. 